\title{
Description of Work Processes Used by Clinical Nurse Specialists to Improve Patient Outcomes
}

\author{
Janet S. Fulton PhD, RN, ACNS-BC, FAAN ${ }^{1}$ \\ Professor \\ ${ }^{1}$ Indiana University School of Nursing \\ 600 Barnhill Drive \\ Indianapolis, IN 46202 \\ jasfulto@iu.edu \\ Ann Mayo DNSc, RN, CNS, FAAN ${ }^{2}$ \\ Professor \\ ${ }^{2}$ University of San Diego Hahn School of Nursing \\ 5998 Alcalá Park \\ San Diego, CA 92110 \\ amayo@sandiego.edu \\ Jane Walker, $\mathrm{PhD}, \mathrm{RN}^{3}$ \\ Associate Professor \\ ${ }^{3}$ Purdue University Northwest School of Nursing \\ $2200169^{\text {th }}$ Street \\ Hammond, IN 46323 \\ walker@purduecal.edu \\ Linda D. Urden DNSc, RN, CNS, NE-BC, FAAN ${ }^{2}$ \\ Professor Emerita \\ ${ }^{2}$ University of San Diego Hahn School of Nursing \\ 5998 Alcalá Park \\ San Diego, CA 92110 \\ urden@sandiego.edu \\ Corresponding Author \\ Janet S. Fulton \\ Indiana University School of Nursing \\ 600 Barnhill Drive \\ Indianapolis, IN 46202 \\ 317-274-2421 \\ jasfulto@iu.edu
}

Funding: This work was supported by Sigma Theta Tau International, Alpha Chapter, Indianapolis, Indiana.

This is the author's manuscript of the article published in final edited form as:

Fulton, J. S., Mayo, A., Walker, J., \& Urden, L. D. (2019). Description of Work Processes Used by Clinical Nurse Specialists to Improve Patient Outcomes. Nursing Outlook. https://doi.org/10.1016/j.outlook.2019.03.001 


\section{Introduction}

Health care systems increasingly are holding nurses accountable for clinical and fiscal outcomes with many of these outcomes being reportable to regulatory and accreditation bodies as well as to the public. Poor outcomes such as medication errors, patient falls and hospital acquired pressure injuries can affect the financial bottom line of an organization, making it imperative that evidence-based best practices are infused into nursing practice. Practice changes designed to improve patient care are nothing new to nurses. However, the science underpinning identifying, measuring, trending, and reporting outcomes has advanced, becoming both more informative and more complex over the past years. Advanced practice nurses (APNs), because of their preparation with gráduate nursing degrees, are likely selected to contribute to monitoring outcomes as well as to conceiving of and leading important outcome-oriented initiatives in their organizations. Nursing recognizes four advanced practice roles - clinical nurse specialists (CNS), nurse practitioners, nurse midwives and nurse anesthetists. Each of the different APN groups contributes to improved outcomes. However, the work of improving nursing care related outcomes is closely aligned with the CNS role and practice because this APN group historically has led nursing practice initiatives to improve nursing care, safety, and cost across systems of care (Fulton, Mayo, Walker \& Urden, 2016).

Expert clinically-focused nursing leadership for improving care delivery and outcomes is much called for and poorly understood. From the historic Brown Report (Brown, 1948) to the more contemporary Institute of Medicine Reports (Finkelman, 2013) nursing and health care organizations have been calling for greater levels of clinical nursing leadership for improved outcomes. Conceptualized in the 1950s, the CNS role was designed to provide 
expert clinical leadership for advancing the practice of nursing practice and improving clinical outcomes (Fulton, 2014). CNSs, a relatively small workforce, are specifically prepared to fill the need for clinical leadership by providing direct care, teaching, and mentoring bedside nurses, and facilitating multidisciplinary care teams and programs at the system level. Of the nation's estimated 3 million nurses, approximately $72,000(<0.3 \%)$ are CNSs (U.S. Department of Health and Human Services \& Health Resources and Services Administration, 2010). Members of the National Association of Clinical Nurse Specialists (NACNS), through research and demonstration projects, have shown that the CNS role is uniquely suited to lead implementation of evidence-based quality improvement initiatives. Some notable areas where CNSs have demonstrated outstanding outcomes include, but are not limited to, prenatal care, behavioral health care, disease prevention, wellness promotion, and all manner of care for persons experiencing chronic illnesses. CNSs make essential contributions to care coordination and care transitions resulting in reduced hospital length of stay and fewer hospital readmissions. Numerous studies have also documented the outcomes of CNS practice in preventing hospital-acquired complications in acute care settings (NACNS, 2014). Additionally, CNSs have been instrumental in supporting health care organizations in achieving and maintaining American Nurses Credentialing Center's Magnet designation status for outstanding nursing services (Walker, Urden \& Moody, 2009; Muller, Hujcs, Dubendorf, \& Harrington, 2010).

There have been several efforts to describe the nature of CNS practice outcomes (Lewandowski \& Adamle, 2009; Mayo et al., 2010). Urden (1999) defined CNS outcomes as the end result or consequence of a nursing treatment or intervention. Oermann and Floyd (2002) identified four categories of treatment outcome - clinical, functional, cost and 
satisfaction. CNS core practice competencies were identified (NACNS, 2004) and validated through research by an expert external review panel (Baldwin, Clark, Fulton, \& Mayo, 2009). Competency linked outcomes of CNS practice have been described (NACNS, 2004) and validated (Fulton, Mayo, Walker, \& Urden, 2016). The original and subsequent updated core competencies define the theoretical and scientific knowledge-based skills needed by CNSs to work effectively in the role and are organized by three domains - patient/client, nursing and nursing practice, and systems/organizations.

Despite a long history of CNS practice, little is known about the specific processes used by CNSs to improve clinical outcomes and promote quality care, safety, efficiency and cost effectiveness. These CNS work processes rely on graduate preparation in the CNS role, clinical practice expertise, and leadership skills for complex systems. Additionally, these CNS work processes are highly influenced by system factors known to effect CNS practice, such as employer understanding of the role, having an office located on the unit where the CNS worked, having a job description, having a framework to guide CNS role development, and having an annual evaluation (Kilpatrick, Tchouaket, Carter, Bryant-Lukosius, \& DiCenso, 2016). Interestingly, CNSs working in differing settings and with different patient populations nonetheless achieve similar types of outcomes. Greater understanding of how CNSs achieve outcomes could provide insight into mechanisms for improving health care. This understanding is particularly relevant in our current era of health care volatility that demands improved clinical outcomes, judicious resource utilization and fiscal conservatism. The aim of this study was to compile a rich description of outcomes-oriented work processes from CNSs with different practice specialties working in variety of practice settings.

\section{Purpose}


The purpose of this qualitative descriptive study was to identify common processes used by clinical nurse specialists (CNS) working in a variety of practice settings and specialties to advance nursing practice and achieve improved clinical outcomes.

\section{Methods}

Design: Qualitative descriptive methods described by Sandelowski (2000) were used to identify the elements of how CNSs work and processes used by CNSs in implementing clinical improvement projects. CNSs are guided by professional practice competencies, therefore it is logical to assume that CNS work experiences have common meanings and associated behaviors, and that shared elements of the work process can be explored through the descriptive method. The co-investigators on this study have a combined total of over 80 years of CNS practice and leadership, as well as teaching CNS students. In this context, along with an identified gap in the literature, the idea for the study emerged. Human subject institutional review boards of the investigators' universities provided study oversight.

Participants and Setting: A purposive sample was used to recruit and assure a diverse group. Participants were selected from among National Association of Clinical Nurse Specialist Conference juried abstract presentations describing project-related outcomes. It was believed that the abstract authors, having submitted their work for public presentation, would be willing to discuss how they achieved the outcomes. Each abstract author was recruited via e-mail. Those interested respondents received an explanation of the study. CNSs agreeing to participate were sent an invitation to a focus group meeting.

Procedures: Participants attended one of two 60 minute focus groups. The purpose of the study was reviewed and participants verbally consented, as had been determined by the 
Human Subjects Committee. Participants were reminded that participation was voluntary and they could leave at any time, though no one opted to leave. A funnel questioning approach was used; the most open-ended question was asked first. Participants shared stories about the process of achieving project outcomes, focusing on the thinking and decision making that guided them in working though the project. Participants were reminded not to discuss the project outcomes, only the processes used to achieve the outcomes. Responses were probed for details about decisions and actions; similarities and difference in experiences were explored. Discussions were audio-recorded and transcribed verbatim; transcripts were reviewed for accuracy.

Analysis: Qualitative descriptive methods were used to analyze the narratives. Qualitative description can be used to obtain a rich description of the common experiences of a group to obtain straightforward answers to questions (Sullivan-Boyai, Bova, \& Harper, 2005). Moderated structured interview with low-inference content analysis, all of which were used in this study, are common in qualitative description (Sandelowski, 2000).

A standard content analysis process (Neuendorf, 2002) was used. The team members read the transcripts several times. Transcripts were then divided among the team members and relevant sections (text units) were coded independently. The text units were compared and contrasted and grouped into subcategories independently. Together team members revised and labeled the subcategories. The quality of the findings was enhanced by use of all researchers to code and categorize data and a consensus procedure (documented by audit trail) was used to resolve discrepancies (Miles, Huberman, \& Saldana, 2014). Demographic data were collected and analyzed using descriptive statistics.

\section{Findings}


Seventeen CNSs participated ( 8 and 9 in each focus group); average age 49.6 years (range: 35 to 62 years); average experience of 24 years (range: 11 - 39) as a registered nurse; average 8.6 years (range: 1.5 to 23 ) working as a CNS; $82 \%$ held master's degree, and $18 \%$ had a doctorate (DNP or PhD). Areas of specialty practice included 53\% adult, $24 \%$ acute/critical care, $6 \%$ perinatal, $6 \%$ pediatrics, $11 \%$ other. Fifteen participants $(88 \%)$ held advanced-level certification including adult (35\%), acute/critical care (18\%), wound/ostomy $(6 \%)$, and $6 \%$ other.

Findings involved descriptions of CNSs engaged in intricate interactions across a diverse workforce of health professionals, administrators, and other workers with varying expertise and interests. The CNS carefully balanced a long term goal with more immediate challenges. The descriptions included detailed accounts of how the CNSs moved a project forward from initial problem identification to the endpoint of success, sustainability and disengagement. Several work descriptions emerged from the findings.

\section{CNS Work Processes for Situating Projects in Context}

Situating Work emerged as an overarching process for fitting the work to context, as each unit or division in an organization was a unique context requiring individualized adaptations. Situating work included processes of beginning with the end in mind, garnering resources, aligning evidence/data, managing tasks, tailoring strategies, managing teams, developing skills in staff, energizing forward movement, monitoring progress, and removing barriers/meeting the challenges of resistance. The multiple elements involved in situating york were noted to be time consuming yet critically important. Processes for situating work are summarized in Table 1. 
Beginning with the end in mind was described as envisioning the outcome, being practical in project scope, and considering sustainability. Envisioning was considered by participants to be purposefully bearing in mind the intended clinical and fiscal outcomes at the patient and system level while crafting a manageable project with a realistic probability of sustainability. One participant stated: I think the role of the CNS is to create that vision. And if you [CNS] can create that vision, the staff can create the details.

Garnering resources was described as projecting resource needs and strategies for obtaining initial and ongoing support. Resources included personnel, access to services, and money. Participants also noted that their time was a resource, and it was important to negotiate for a manageable number of assignments and projects. One participate explained: I'm very protective of not getting pigeon-holed into certain tasks that I have to do constantly... things that really eat up our time. One caution offered by a participant was: ... you have to be a little bit sensitive because people will ask you to participate in their project and you don't want to burn bridges because you need them in the future.

Aligning evidence and data was reported as a process of securing evidence and data that provide shape and dimension to the problem. Data were described as necessary for problem identification and for ongoing monitoring and evaluation. One participant explained: I spend a fair amount of time figuring out what data is available... and if it isn't available, how can I create it. I will need that data on the backend to demonstrate project outcomes. Data were also noted to be important because perceptions of health care providers are often inaccurate. One participant stated: We were instituting a sepsis protocol... so we went to the critical care medical staff and asked what they thought about it. We went there thinking of course they'll see [need for the protocol]. And of course, they didn't. Every 
single one of them shot it down because they said we do this already, we don't need a protocol. We were flabbergasted. So we did a chart review that clearly indicated those things were not being done. We went back to the group and every single one of them completely turned around and said we were right, we needed a protocol.

Managing tasks was described as being exquisitely sensitive to the work environment and organizational culture. Suggested strategies for managing tasks were breaking the project work into small steps or tasks and working from small to large accomplishments along a trajectory that culminates in project completion. Conducting small pilot tests and monitoring the work as it unfolds were suggestions for keeping the project controlled and allowing for immediate adjustments to changing circumstances. One participant noted: ...you have to really sit back and even though you'd like to go a little faster you have to sit back, get feedback...

Tailoring strategies, described as adapting change strategies to organizational demands, unit cultures, and human and fiscal resources, was considered very important to project success. Participants gave many examples of how the same project would require unique approaches, team members, and timelines when implemented for different units or in other settings. As one participant noted: ...there's unit culture, but then you can have cultures within cultures... Another participant stated: ...it does boil down to understanding workflow in the hospital and how different interdisciplinary teams come together.

Managing the team was described as working responsibly and respectfully with a variety of people, finding the right person for each of the various aspects of the project work, and keeping the team connected to the whole project. The participants also noted that team management included managing interpersonal dynamics in situations like personnel changes, 
such as losing or adding a team member, and making hard choices when the team loses focus.

Supporting staff skill development was described as important in preparing staff to engage in the work of the project and in sustaining the change time. Participants agreed that, depending on the project and setting, it may be necessary to provide education, training or skill development. Skills such as chart audits, library searches, literature reviews and conducting SBAR - situation, background, assessment, recommendation, are often necessary to support projects. Skill development may be needed initially, as the start of the project, or ongoing as the work proceeds, team members change, and staff members turn over. One participant, finding that she was not achieving intended outcomes, stated she had to reeducate the staff on proper performance of the new procedure.

Energizing forward movement was described as leading a team through the project journey. This work required multiple strategies such as being involved when others resist, persisting in the face of resistance, being mindful of change overload, and keeping the team engaged. One participant stated: My role in moving it forward was working behind the scenes with all the other disciplines and continuously getting feedback from the staff while coaching them along. Another participant stated: It's coaching and mentoring, so the staff own the process... and it just creates a whole different energy than just being told this what you're gonna do.

Meeting the challenge of resistance was described as implementing strategies for overcoming barriers to moving a project forward. Challenges, occurring at any time in the project initiative, was a pervasive topic among participants, and they shared many examples related to overcoming barriers. Barriers that participants noted included regulatory 
requirements, reimbursement rules, organizational politics, and staff resistance, especially from nurses and physicians. Participants noted the importance of finding the reasons for resistance and addressing concerns openly. Interpersonal barriers that made open discussion difficult included being ignored, being avoided, I'll get back to you, and being dismissed, I don't have time for that. About resistance, one participant stated: It's easy to figure out when they are saying yes. It's harder to figure out when they're saying no. Cautious persistence in the face of barriers was frequently advised. One participant stated: I think one of the talents a CNS brings to the table is recognizing that it's not a barrier, it's not an end stop, it's called rerouting...to overcome, to renegotiate, to find the right fit for whatever is in front of me so that I keep this project moving, sustain this initiative, and produce the outcome we're looking for.

Monitoring progress was described as the continual evaluation of project progress and making adjustments in tasks and resources as needed. Monitoring during the project presupposed the outcome to be achieved was established ahead of time and the measures are in place to track progress. Participants agreed that monitoring should be continual throughout project implementation and after completed. A participant explained: I think one of the biggest mistakes we made after we implemented a project is to turn our attention to something else and not give continual monitoring its due. Dashboards, score cards, quality audits, fiscal reports, and many other strategies were described for monitoring progress and sustainability.

\section{CNS Work Processes for Achieving Project Outcomes}


Study participants described using multiple processes to achieve project outcomes. These processes with examples of associated activities are summarized in Table 2 and described in more detail below.

Identifying a Problem was a predictable first step in achieving a project goal. Participants stated that problems were brought to their attention in many different ways, including being called by the staff to solve a problem, being assigned by administrators, and applying their CNS knowledge and expertise to making clinical rounds, reading administrative reports, and receiving requests for assistance. They reported the importance of listening and observing - listening for problems and observing changes in care practices and outcomes. One participant noted: You need to be out there, not in your office, you need to hear what the concerns are. Another participant stated: I've been most successful with problems identified by staff nurses... they notice a difference in practice and ask about doing something to fix or standardize care. Participants cautioned that the problem should be validated before taking action. One participant stated: We knew there was an issue, but we got data to help us confirm... and decided there were two layers of related problems.

Engaging Stakeholders involved the work processes of identifying stakeholders, conducting preparatory steps for engagement, and onboarding stakeholders. Overall this was a multi-staged, complex, and layered process. Identifying stakeholders required time and finesse. Persons with an interest were not always apparent, and participants described the importance of engaged conversations to uncover vested interests and competing agendas. In addition, many obvious stakeholders needed convincing that the problem was a problem. Participants reported they influenced the stakeholder's perceptions of the problem by using data, probing questions, and storytelling. One participant explained: How you do it is 
different, depending on who you're finessing... use different approaches to get them where you need them to go. Participants noted the importance of identifying and engaging champions for the project, especially among the staff nurses.

Forecasting involved reading situation dynamics and seeing possibilities. Participants noted the importance of continuously interpreting the contextual dynamics of the environment by picking up cues, making adjustments, and troubleshooting. One participant explained: Sometimes you have to sit back... adjust the pace...get feedback, feel it out... you have to feel the pulse of the unit. Participants also noted the importance of being open to new possibilities, recognizing limitations, and making adjustments and revisions. One participant stated: You find out as you start delving in... you have to stop and restart... you find things you didn't even think about. Another participant noted: ....sometimes there's these good ideas just sort of pop up. Overall, participants viewed adjustments and revisions as a normal part of the process, even when delays were long and obstacles were challenging. Their desire to achieve the goal was the driving force. One participant summed this process as: You start in a direction, you may be rerouted, but know what you want to achieve.

Providing Feedback was described as a critical work process for project success that occurred through communicating, providing support and encouragement, and seeking feedback from others. Continuous feedback to nurses, project team members and others was described as essential for keeping everyone engaged as the project moved forward. One participant stated: Having constant feedback was very important for front line staff, all the team members and their departments. Participants noted the importance of also seeking feedback from others by asking for feedback, consulting with colleagues, and seeking external review. Feedback was described as being respectful and collegial. One participant 
stated: It's been about two years with this one intensivist physician, and last week for the first time, I thought I'm finally getting there. I show him data, show him respect, show him data, show him respect, show him data... its persistence.

Interfacing with the System included processes of connecting with administrators, bridging interdisciplinary teams, and connecting within the system. Connecting with administration was described as deliberately communicating with system administrators, negotiating priorities with administrators, and seeking administrator counsel when a broader perspective was needed. Staying connected within the system involved serving on interdisciplinary committees and being involved in system-wide initiatives. One participant stated: CNSs need to stay at the table, be on the committees.. not just nursing committees but physician and hospital committees... so you have the opportunity to share. Bridging interdisciplinary teams was described as being able to maneuver around organizational politics and culture and coordinate multiple agendas. Participants considered it very important to understand the role of other team members, to build relationships with formal leaders, and to know and cultivate relationships with informal thought leaders. One participant stated: There are different kinds of leaders, and different issues and challenges.

Disseminating reports throughout the system was described as both necessary and challenging. Reporting was considered vital to helping others in the system understand how a CNS-led project contributed to improved outcomes and, ultimately, the organization' s mission, but also to recognizing the work outcomes of CNS practice. Participants described regularly disseminating their findings to hospital quality committees, medical staff council, and the executive board. Participants stated that their reports included cost-savings whenever possible to demonstrate contributions to the fiscal bottom line. One participant stated: Once 
a year we do a presentation for the medical staff council and executive board...we feature one major CNS-led project with quality measures and cost savings. Another participant, noting that she had been unsuccessful in finding an appropriate system-wide forum in which to present quality reports, stated: I started publishing a PowerPoint with about ten different charts of data that I thought were important... and I send it to the administrators of our division every month. She stated that after 2 years of sending the data, someone invited her to serve on the hospital's quality committee.

\section{Having Self-Agency}

Embedded in the accounts of the project implementation processes were insights into the professional attributes that helped the CNS participants link their practice competency to overall effectiveness. Having a sense of self-agency grounded in one's own professional attributes emerged as foundational to participants"ability to function in the CNS role. Having self-agency was expressed byparticipants in descriptions of taking responsibility, owning nursing practice, influencing others, and maintain self-awareness though reflection.

Taking responsibility referred to participants agreeing that, within the system, they were accountable for addressing clinical nursing problems and leading practice improvement changes. They acknowledged that a team effort was necessary to achieve change, but saw themselves as the vanguard for advancing practice in their specialty area. One participant stated: Ifelt this obligation and responsibility... to look deeper into why our patients were getting so many pneumonias and what we could do. Other participants described themselves as the person who takes the initiative, steps up to the plate, and follows through to fix clinical problems. One participant, clearly noting that she was holding herself responsible for patient outcomes, stated: It was my responsibility, or my self-inflicted responsibility I guess 
you would say, to make sure that all those processes were working and that nursing made a difference for those patients.

Owning Nursing Practice was apparent through participants' keen sense of ownership of nursing practice. They discussed the importance of CNSs articulating and demonstrating nursing's autonomous authority to practice. This notion of owning nursing's autonomous practice authority in a team practice environment was highlighted by one participant who stated: My project was about nursing practice, basic nursing practice. Did I need any physician's order for it? No. Did I really need them to be part of it? No, but did I have them on my team? Yes. Did I keep them in the loop on data? Absolutely. Did I go report to their group meetings and grand rounds and tell them what nurses are doing? Absolutely. I didn't want to exclude, but we didn't really need their permission. Owning nursing practice included descriptions of needing to maintain control of the project and being able to take credit for the outcomes. One participant stated: The physicians do watch what we do, and once they get on to what's a good thing, they want to hijack it... take over our practice.

Self-Reflecting was described by participants as the importance of being able to manage themselves, or have self-control, and was viewed as critical to their professional performance and project success. Processes like finessing people, navigating barriers, and managing resources required high levels of self-reflection. Participants described a general inclination to take on too much and say "yes" to too many initiatives. Self-reflection helped them manage themselves and therefore the work by staying focused, managing time, dealing with delays, and prioritizing. Self-reflection was important for navigating interpersonal challenges and political agendas. One participant noted: You have to take a step back and 
prioritize. It's really hard because to everyone else their project is the most important project.

For many participants, self-reflection was a way of validating their role, acknowledging their abilities, and managing self-doubt. Participants described feeling really inadequate when a project did not go as planned. They described coming to an understanding that it was acceptable to fail sometimes and to use failure as a learning opportunity. One participant explained: ... I was thinking I needed to put something out there right away and it needed to be perfect the first time, and when it wasnit I'd get really upset with myself... until accepting and learning from it. Self-reflection also helped them recognize that, in spite of competence and expertise in many areas, there are times when it may be necessary to seek help in some areas.

\section{Influencing Others}

Self-agency underpinned the CNSs participants' confidence and therefore ability to influence others. All participants worked in supportive staff positions without managerial authority. Their ability to implement change rested largely on the ability to influence and motivate. One participant noted: You're not a direct supervisor... and in a way that makes it harder because I have to get people to do things that I would like them to do without any direct authority. Participants noted that influencing occurred through sharing, role modeling, coaching, and mentoring. These influencing strategies required presence - the CNSs had to be present in clinical areas and available to staff as questions arose and situations unfolded. Maintaining presence was described as time consuming and necessary. One participant stated: ... half the job is a sales job... getting them to want to do it.

\section{Building Trust}


Participants spoke at length about the importance of trust - building trust, using earned trust, and gaining trust capital. Trust was central and necessary for project success. Participants described the need to build trust as a first step in project work. One participant explained: If you don't start off trusted it's hard to move a project forward. Strategies for building trust included showing respect, appreciating difference, listening, and collaborating. Being able to build trust was described as critical to a new CNS in any clinical setting. One participant suggested building up a reputation of trust through a series of small successes focusing on low hanging fruit because, as she explained... they need to trust you, the physicians and nurses... you can't just go in there and say 'okay, this is how we are doing it.'

Using earned trust is glue that holds the project implementation together. Participants stated that staff would work on a project because they trusted the CNS to be positive, to get the job done, and to keep the staff safe during the process. Several participants noted that staff view the CNS as a safe confident, and they told stories of using trusting relationships to help staff work though difficult situations, One participant stated: Staff come to the CNS to confide... we're not management, we're someone they can talk to... who is not going to be punitive and will give them advice.

Gaining the trust of persons across the system is a form of trust capital that can be banked and used in the future. Participants explained that trust capital happens by spending time on committees, in ad hoc work groups, with interdisciplinary colleagues working on their projects, sharing credit for success, and maintaining positive working relationships even through difficult circumstances. Working across the system is a good opportunity for banking trust capital; however, several participants noted challenging consequences of trust. One explained: The chief safety officer pulls me into his initiatives, he trusts me. But I have 
to balance the gains I get from working with him and the other demands on my time.

Participants all agreed that trust was foundational to CNS practice. One participant summed up the trust topic by saying: ...it's real important in our role to keep that openness so we're a safe, trusted role model.

The relationships among the work processes used by CNSs in conducting a clinical change project are summarized in Figure 1. Initial and ongoing work processes situate the project in the unique context. The project moves forward in a loose, stepwise flow including identifying the problem, engaging stakeholders, forecasting, providing feedback, interfacing with the system, and disseminating. Self-agency is essential to the CNS role, and is reflected in the CNS owning nursing practice, taking responsibility for solving problems and advancing practice, influencing others to achieve goals, and marinating balance and selfcontrol through reflection. Self-agency leads to trust - building trust, using trust and gaining trust capital.

\section{Discussion}

Clinical nurse specialists are responsible for identifying clinical problems and initiating system-level interventions to prevent or improve management of the problems (Whitman \& Pervis, 2015). CNS practice competencies include leading change for improved clinical outcomes (Fulton, et al., 2016). This study provided insight into how CNSs practice and the work processes used by CNSs in achieving clinical outcome improvements. The patient-centered activities of CNS have been studied (Norton, Sigsworth, Heywood \& Oke, 2012), the activities related to system-level clinical leadership have been described (Elliott et al., 2012), and the importance of CNS leadership for the sustainability of a project has been 
evaluated (Babine, Honess, Wierman, \& Hallen, 2016). However, these studies provided limited insight into how CNSs work.

We analyzed descriptions of the processes CNSs used to complete a system-level project. For the purpose of this study, a project was considered a significant undertaking involving a collaborative enterprise that was carefully contemplated, devised and planned. The descriptions captured a project as an unfolding narrative across time. The projects had diverse elements and varied in scope, intensity, and structure. All projects described occurred at the system-level and involved multiple providers and stakeholders. The descriptions revealed an uneven project course with periods of delay and revisions. The impetus for the projects varied; some were self-initiated in response to gaps in care, and others were administratively identified and handed off to the CNS as a work assignment. In all cases, the project was the work of the CNS.

Work is a vague concept, though generally understood to be the effort exerted to accomplish a task or goal. Work is valued. The value of work can be both shared and individualized. In the context of employment, work and resulting outcomes are completed for compensation. The actual doing of the work can be standardized across individuals in an employment setting with emphasis on the tasks of the work, or it can be highly individualized with emphasis on the outcome of the work. Professional work, such as nursing, medicine, or law, is referred to as practice. Examining work related tasks can be an approach to examining professional activities, progress and outcomes; however, examining work roles is more related to the underlying context of tasks and form the basis for shared purpose, meaning and values (Huvila, 2008). In this study, many and varied individual tasks, or actions, were described by the CNS participants, yet when performed in the context of the 
CNS role formed a shared purpose and value that gave insight into the CNS role. Thus the process work identified in this study can be considered CNS practice.

Work is context dependent. The contextual system in which work exists creates the state for establishing and reestablishing the work itself (Huvila, 2008). Participant descriptions aptly noted the need to contextualize the project work. Contextualization, as seen in the descriptions, occurred as a first effort and reemerged as needed during the project implementation. Participants described the need to be ever-vigilant to context changes. Context was described by participants as having static elements, such as organizational mission and physical facilities, and fluid elements, such as material resources and staff skill levels. Being able to successfully manage a project was identified as the process of being exquisitely sensitive to the work environment and organizational culture.

Work is related to control. Control implies the ability to guide and coordinate work and is an important force that permits work to emerge (Suchmann, 1995). Controlling work involves coordinating the organization and management of multiple elements to establish efficiency and allow the work to exist. This coordination activity has been referred to as articulation work (Strauss, 1988). Strauss originally developed the notion of articulation work to describe the small activities, or micromechanics, of social situations seen in customer service workers.

Currently, the notion of articulation work is viewed largely through the lens of computers and information systems, where computer support facilitates articulation work by providing a way to steer and connect interdependent task activities in a field of work. Computers may help with articulation of assembling, scheduling, monitoring, and coordinating all of the steps necessary to complete a production task. However, older 
sociological studies looked at articulation work as behind the scenes work performed invisibly and of great importance to the achievement of formal work outcomes. For example, Jackson (1997) examined routine prescribed care for nursing home residents and found that non-routine or personal care, such as bringing a card or flowers, taking residents for a walk, setting up a talking book program, or putting on a patient's glasses for her, was of great importance to residents. This caring work used the individual care-worker's knowledge and judgement to articulate the daily care routine for the residents, which contributed to overall patient satisfaction with care. This type of work is performed largely in context and within relationship. Articulation work has also been described as imbedded in social situations and largely invisible (Hampton \& Junor, 2005).

Articulation work involves coordination and integration. Articulation work involves working through and carrying out work-related arrangements (Corbin \& Strauss, 1993). It is the meshing of numerous tasks, clusters of tasks, segments of work, and the efforts of multiple workers with differing skills into an organized and meaningful effort. Articulation work has been described as the unacknowledged management of awkward intersections among the social worlds of people, technology and organizations (Hampton \& Junor, 2005). Strauss (1985) noted that projects proceed along an "arc of work" and articulation work integrates segmented efforts into the total arc of the project. The essential point behind the notion of articulation work is a cognizant awareness of what is being done in a collaborative activity (Gerson and Star, 1986). This study demonstrated that the work of achieving outcomes through a system-level project overwhelmingly involved articulation work as participants provided multiple examples related to managing the tasks of the project (See Table 2 for descriptions of articulation work activities). 
Central to project work is the notion of interactional processes, which is considered essential to accomplishing both articulation work and to getting the work done despite the inevitable interruptions and impediment to work flow (Strauss, 1988). Interactional processes are different from overall work tasks and include things like persuading others, demonstrating value, negotiating, tailoring the message (Strauss, 1988). Examples of participant descriptions of actions such as giving praise, listening to problems, smoothing things out, and negating priorities demonstrate the high level of interactional processes used by the CNSs in project work. Multiple participants described the need to tailor messages and approaches to differing stakeholders.

Articulation work is largely invisible. Visible articulation work is classic management work, and participants described some standard management tasks such as preparing reports, assessing staff skill levels, and developing protocols. However, many participant descriptions involved invisible artículation, which involves recognizing and buffering stress and change, responding to contingencies, dealing with unexpected conditions, adapting and adjusting in response to unanticipated events (Hampton \& Junor, 2005). The concept of invisible work was created to bring sociological attention that work that was unrecognized or undervalued (DeVault, 2014). Nurse work has been noted to be invisible work, often taken for granted as disembedded background work (Star \& Strauss, 1999).

Should, or could, invisible work be made more visible? Wears (2012) noted three possible pitfalls to any efforts to make the invisible visible. First, there is the risk that only the visible work will be identified. Expert work is difficult to see, and the more expertly it is done, the less visible it is. Second, making the invisible visible renders it amenable to 
external control and manipulation. Invisibility in itself is a source of control enabling expert workers to control how they work and how the work is expressed. Autonomy is a hallmark of professional practice and as such relies on internal controls to be effective. Third, lacking established methodologies, simple descriptive studies will not result in unpacking activities in a meaningful way. Work tends to diminish and simplify when viewed from a distance (Suchman, 1995). CNSs have autonomy and authority over personal and professional knowledge and experience suggesting these professionals have the ability to shape not only the work but how the work appears to others. Self-representation is a form of empowerment (Suchman, 1995).

Empowerment gives a person the authority to do something. Participants in this study provided rich descriptions of empowerment through self-agency. The participants described themselves as taking responsibility for and owning nursing practice. They described using measured self-reflection to maintain balance and perspective. A foundation of self-agency facilitated their ability to achieve outcomes largely though influence. Influence was critical to CNS work processes because they lacked management authority and relied completely on influencing in the context of collaborative relationships.

Influence, as practiced in nursing, is poorly understood. Adams and Natarajan (2016) noted that acquiring influence, and knowing how to use it, is a competency for nurse leaders. Within their model, the Adams Influence Model (AIM), power is defined as the cumulative result of being able to influence multiple issues, and is based on relationships and intentionality in a leader's efforts to shape the care environment and effect change. The CNS participants in this study relied on influence to drive change and complete a project. CNSs 
long have been recognized as powerful influencers of clinical care in an organization. The findings of this study reinforce the importance of influence used well.

Trust emerged as central to all CNS work processes. Trust was described as a type of capital that can be earned, banked, spent, and lost within work relationships. Gaining the trust of others was described as essential and something to be attended to vigorously. Participants described purposeful actions taken to engender trust. Gilbert and colleagues (2017) noted that trust was the most commonly described attribute of social capital within an organization. The findings of this study suggest that self-agency, trust, and influence are a nexus upon which CNS work processes revolve.

\section{Limitations}

Participants were from North American health care systems (USA and Canada), and while structurally different they are similar in the level of health care delivered, thus the findings may not reflect CNS processes used in project work occurring in other systems in different countries. Participants were recruited from among those CNSs reporting successful improvement project outcomes, therefore little can be deduced about processes that were not successful.

\section{Conclusion}

The findings of this study provide insight into CNS work processes, providing opportunity to better understand the nature of CNS practice and lending credibility to CNS leadership abilities. CNS work processes can be described as predominately articulation work involving the management of intersections among the social worlds of people, technology, and organizations. Articulation work is invisible resulting in the work being unrecognized or undervalued. As CNSs become more and more engaged in applying 
knowledge and expertise to system-level projects in health care, the more invisible they become. This study provides insight into the very real but often invisible work processes used by CNSs in supporting and leading healthcare teams and improving outcomes, and helps explain why the CNS role and practice is often considered invisible and ambiguous.

Regardless of country, CNS-led clinical improvement projects are central in advancing nursing practice and improved outcomes. CNS practice is situational and contextual; processes used by CNSs to gain trust, solve problems, and communicate with other disciplines are essential to CNS practice. CNS education should incorporate these processes into curricula; new CNSs should be mentored in mastering these competencies and developing self-agency. Administrators need to support these critical, though often invisible, CNS work processes because CNS-led improvement initiatives will enhance the CNS role across specialties and settings, support individual CNS job satisfaction, and ultimately improve patient care outcomes.

Future research should provide closer examination of clinical and fiscal outcomes of CNS practice related to improvement projects. Also, studying this phenomenon in other countries where CNSs practice might validate the findings in this study, or reveal other unique characteristics that foster CNS success in achieving health care outcomes 


\section{References}

Adams, J.M. \& Natarajan, S. (2016). Understanding influence within the context of nursing: Development of the Adams influence Model using practice, research, and theory. Advances in Nursing Science, 39(5), E40 - E56.

Babine, R.L., Honess, C., Wierman, H.R. \& Hallen, S. (2016). The role of clinical nurse specialists in the implementation and sustainability of a practice change. Journal of Nursing Management, 24, 39 - 49.

Baldwin, K.M., Clark, A.P., Fulton, J. \& Mayo, A. (2009). National validation of the NACNS clinical nurse specialist core competencies. Journal of Nursing Scholarship, 41(2), 193-201.

Brown, E.L. (1948). Nursing for the future. New York, NY: Russel Sage Foundation.

Corbin, J.M \& Strauss, A.L. (1993). The articulation of work though interaction. The Sociological Quarterly, 34(1), 71-83.

Elliott, N., Higgins, A., Begley, C., Lalor, J., Sheerin, F., Coyne, I. \& Murphy, K. (2012). The identification of clinical and professional leadership activities of advanced practitioners: findings from the Special Clinical and Advanced Practitioner Evaluation study in Ireland. Journal of Advanced Nursing, 69(5), 1037-1050.

DeVault, M.L. (2014). Mapping invisible work: Conceptual tools for social justice projects. Sociological Forum, 29(4), 775-790.

Finkelman, A. (2013). The clinical nurse specialist: Leadership in quality improvement. Clinical Nurse Specialist, 27(1), 31-35.

Fulton, J.S. (2014). Evolution of the clinical nurse specialist role and practice in the United States. In J. S. Fulton, B. L. Lyon \& K. A. Goudreau (Eds.). Foundations of clinical nurse specialist practice ( $2^{\text {nd }}$ ed., pp. 1-15). New York, NY: Springer.

Fulton, J.S., Mayo, A.M., Walker, J.A. \& Urden, L. D. (2016). Core practice outcomes for clinical nurse specialists: A revalidation study. Journal of Professional Nursing, $32(4), 271-282$.

Gerson, E. \& Star, S.L. (1986). Analyzing due process in the work place. ACM Transactions on Office Information Systems, 4(3), 257-270.

Gilbert, J.H., Von Ah, D. \& Broome, M.E. (2017). Organizational capital and the role of the nurse manager: A proposed conceptual model. Nursing Outlook, 65, 697-710. 
Hampton, I. \& Junor, A. (2005). Invisible work, invisible skills: Interactive customer service as articulation work. New Technology, Work and Employment, 20(2), 166181.

Huvila, I. (2008). Work and work roles: A context of tasks. Journal of Documentation, 64(6), 797-815.

Jackson, E.M. (1997). Dimensions of care in five United States nursing homes: Identifying invisible work in care-giving. International Journal of Nursing Studies, 34(3), 192200 .

Kilpatrick, K., Tchouaket, E., Carter, N., Bryant-Lukosius, D., DiCenso, A. (2016). Structural and process factors that influence clinical nurse specialist role implementation. Clinical Nurse Specialist, 30(2), 89-100.

Lewandowski, W. \& Adamle, K. (2009). Substantive areas of clinical nurse specialist practice: A comprehensive review of literature. Clinical Nurse Specialist, 23(2), 7390.

Mayo, A, Omery, A., Agocos-Scott, L.M., Khaghani, F., Meckes, P.G., Moti, N., Redeemer, J., Voorheers, M., Gravell, C. \& Cuenca, E. (2010). Clinical nurse specialist practice patterns. Clinical Nurse Specialist, 24(2), 60-68.

Miles, M.B., Huberman, A.M. \& Saldana, J. (2014). Qualitative Data Analysis: A Methods Sourcebook. Washington, D.C.: Sage.

Muller, A.C., Hujcs, M., Dubendorf, P.\& Harrington, P.T. (2010). Sustaining excellence: Clinical nurse specialist practice and magnet designation. Clinical Nurse Specialist, 24(5), 252-259.

National Association of Clinical Nurse Specialists (2014). Impact of the Clinical Nurse Specialist Role on the Costs and Quality of Health Care. Available at: https://nacns.org/advocacy-policy/position-statements/impact-of-the-clinical-nursespecialist-role-on-the-costs-and-quality-of-health-care/ Accessed January 5, 2019.

National Association of Clinical Nurse Specialists (2004). Statement on Clinical Nurse Specialist Practice and Education, Harrisburg, PA: Author.

Neuendorf, K.A. (2002). A Content analysis guidebook. Thousand Oaks, CA: Sage.

Norton, C., Sigsworth, J., Heywood, S. \& Oke, S. (2012). An investigation into the activities of the clinical nurse specialist. Nursing Standard, 26(30), 42-50.

Oermann, M.H. \& Floyd, J.A. (2002). Outcomes research: An essential component of the advanced practice nurse role. Clinical Nurse Specialist, 16(3), 140-144. 
Sandelowski, M. (2000). Whatever happened to qualitative description? Research in Nursing and Health, 23, 334-340.

Star, S.L. \& Strauss, A. (1999). Layers of silence, arenas of voice: The ecology of visible and invisible work. Computer Supported Cooperative Work, 8, 9 - 30.

Strauss, A. (1985). Work and the division of labor. The Sociological Quarterly, 26(1), 1 19.

Strauss, A. (1988). The articulation of project work: An organizational process. The Sociological Quarterly, 29(2), 163 - 173.

Suchman, L. (1995). Making work visible. Communications of the ACM, 38(9), 56-64.

Sullivan-Bolyai, S., Bova, C. \& Harper, D. (2005). Developing and refining interventions in persons with health disparities: The use of qualitative description. Nursing Outlook, $53,127-133$.

Urden, L.D. (1999). Outcome evaluation: An essential component for CNS practice. Clinical Nurse Specialist, 13(1), 39 - 46.

U.S. Department of Health and Human Services \& Health Resources and Services Administration, 2010). The registered murse population: Findings from the 2008 national sample survey of registered nurses. Retrieved from https://bhw.hrsa.gov/sites/default/files/bhw/nchwa/rnsurveyfinal.pdf

Walker, J.A., Urden, L.D. \& Moody, R. (2009). The role of the CNS in achieving and maintaining Magnet status. Journal of Nursing Administration, 39(1), 515-523.

Wears, R.L. (2012). Work, visible and invisible. Annals of Emergency Medicine, 59, 374375.

Whitman, N. \& Purvis, S. (2015). Improving outcomes for high risk patients: Creating timely reports from the electronic health record. Clinical Nurse Specialist, 29(5), 283-289.

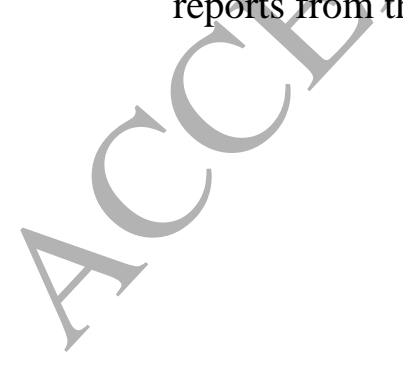




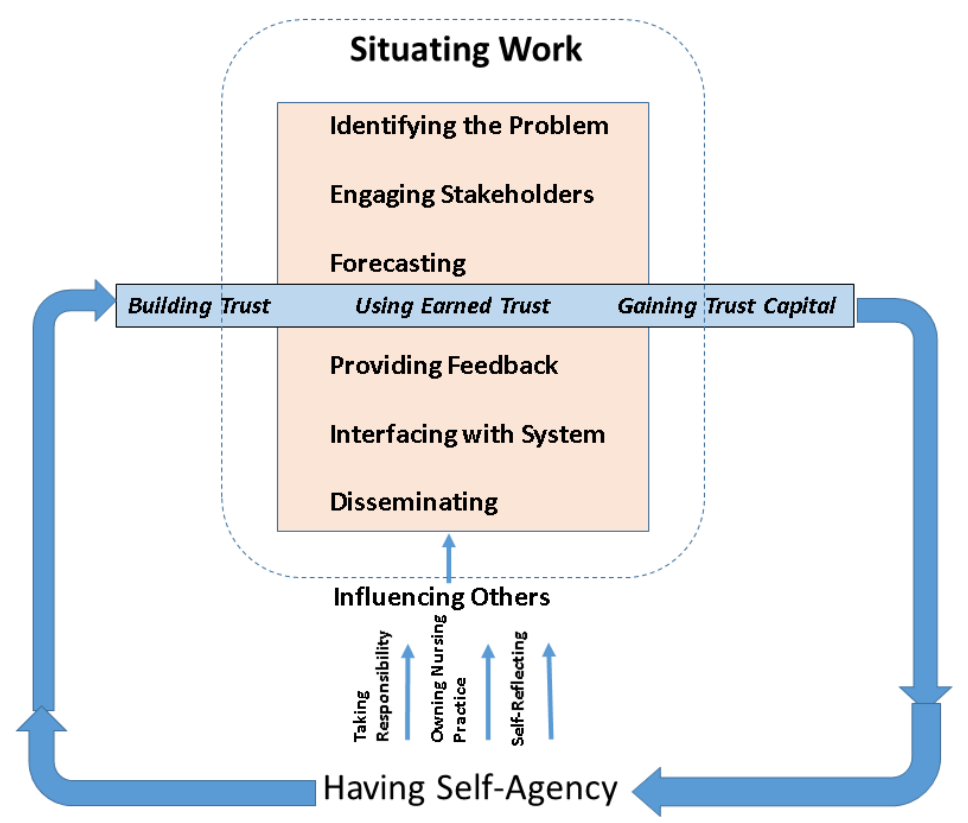

Figure 1: The relationships among work processes used by clinical nurse specialists conducting clinical change projects. 
Table 1: Clinical Nurse Specialist Work Processes for Situating Projects in Context

\begin{tabular}{|l|l|}
\hline \multicolumn{1}{|c|}{ Process } & \multicolumn{1}{c|}{ Definition } \\
\hline Beginning with the end in mind & $\begin{array}{l}\text { Envisioning the outcome, being practical in project scope, } \\
\text { and considering sustainability. }\end{array}$ \\
\hline Garnering resources & $\begin{array}{l}\text { Projecting resource needs and strategies for obtaining } \\
\text { initial and ongoing support. }\end{array}$ \\
\hline Aligning evidence/data & Securing evidence and data that align with the problem. \\
\hline Managing tasks & $\begin{array}{l}\text { Being exquisitely sensitive to the work environment and } \\
\text { organizational culture. }\end{array}$ \\
\hline Tailoring strategies & $\begin{array}{l}\text { Adapting change strategies to organizational demands, } \\
\text { unit cultures, human and fiscal resources. }\end{array}$ \\
\hline Managing teams & $\begin{array}{l}\text { Working responsibly and respectfully with people, aligning } \\
\text { people with tasks, and keeping the team connected to the } \\
\text { whole project. }\end{array}$ \\
\hline Developing skills in staff & $\begin{array}{l}\text { Preparing staff to engage in the work of the project and to } \\
\text { support evaluating and sustaining the change time. }\end{array}$ \\
\hline Energizing forward movement & Leading the team through the project journey. \\
\hline Monitoring progress & $\begin{array}{l}\text { Continual evaluation of progress and making adjustments } \\
\text { in tasks, and resources }\end{array}$ \\
\hline Meeting the challenges of resistance & $\begin{array}{l}\text { Implementing strategies for overcoming barriers to moving } \\
\text { a project forward. }\end{array}$ \\
\hline
\end{tabular}


Table 2: Clinical Nurse Specialist Work Processes for Achieving Project Outcomes

\begin{tabular}{|c|c|c|}
\hline Process Category & Process Activities & Examples \\
\hline \multirow[t]{8}{*}{ Identifying a Problem } & \multirow[t]{3}{*}{ Using staff input } & Getting called by staff to solve a problem \\
\hline & & Listening for problems \\
\hline & & Helping nurses bring ideas forward \\
\hline & \multirow{3}{*}{$\begin{array}{l}\text { Using CNS knowledge and } \\
\text { expertise }\end{array}$} & Noticing changes in patient care outcomes \\
\hline & & $\begin{array}{l}\text { Identifying differences by making clinical } \\
\text { rounds }\end{array}$ \\
\hline & & Asking questions in interdisciplinary groups \\
\hline & \multirow{2}{*}{ Engaging with others } & Recruited by a colleague to problem solve \\
\hline & & $\begin{array}{l}\text { Assigned to solve a problem by } \\
\text { administrator }\end{array}$ \\
\hline \multirow[t]{11}{*}{ Engaging Stakeholders } & \multirow[t]{3}{*}{ Preparing to engage } & Collegial dialoguing \\
\hline & & Planting idea seeds \\
\hline & & Gauging responses \\
\hline & \multirow{5}{*}{$\begin{array}{l}\text { Conducting preparatory } \\
\text { steps for engagement }\end{array}$} & Finding champions \\
\hline & & Understanding stakeholder roles \\
\hline & & Using data to demonstrate \\
\hline & & Using storytelling to influence \\
\hline & & Ask probing questions \\
\hline & \multicolumn{2}{|c|}{\begin{tabular}{l|l} 
Onboarding stakeholders & Securing cooperation \\
\cline { 2 - 2 }
\end{tabular}} \\
\hline & & Defining shared vision \\
\hline & & Developing partnerships for mutual benefit \\
\hline \multirow[t]{6}{*}{ Forecasting } & \multirow{3}{*}{ Reading situation dynamics } & Picking up cues \\
\hline & & Making adjustments \\
\hline & & Smoothing things out \\
\hline & \multirow{3}{*}{ Seeing possibilities } & Finding new opportunities \\
\hline & & Recognizing limitations \\
\hline & & Being open to revisions \\
\hline \multirow[t]{9}{*}{ Providing Feedback } & \multirow{6}{*}{$\begin{array}{l}\text { Communicating } \\
\text { Providing support and } \\
\text { encouragement }\end{array}$} & Keeping team engaged \\
\hline & & Clarifying expectations \\
\hline & & Sharing results \\
\hline & & Providing counsel \\
\hline & & Giving praise \\
\hline & & Answering questions \\
\hline & \multirow{3}{*}{$\begin{array}{l}\text { Seeking feedback from } \\
\text { others }\end{array}$} & Asking for feedback \\
\hline & & Consulting with colleagues \\
\hline & & Seeking external review \\
\hline \multirow{8}{*}{$\begin{array}{l}\text { Interfacing with the } \\
\text { System }\end{array}$} & \multirow{3}{*}{$\begin{array}{l}\text { Connecting with } \\
\text { administration }\end{array}$} & Negotiating priorities with administrators \\
\hline & & $\begin{array}{l}\text { Talking with nurse executive when the work } \\
\text { gets tough }\end{array}$ \\
\hline & & $\begin{array}{l}\text { Having hallway meetings with } \\
\text { administrators }\end{array}$ \\
\hline & \multirow[t]{3}{*}{$\begin{array}{l}\text { Bridging interdisciplinary } \\
\text { teams }\end{array}$} & $\begin{array}{l}\text { Maneuvering around organizational politics } \\
\text { and culture }\end{array}$ \\
\hline & & Serving on interdisciplinary committees \\
\hline & & Coordinating multiple agendas \\
\hline & \multirow[t]{2}{*}{$\begin{array}{l}\text { Connecting within the } \\
\text { system }\end{array}$} & $\begin{array}{l}\text { Having a direct line to nursing and hospital } \\
\text { administration }\end{array}$ \\
\hline & & Being involved in system-wide initiatives \\
\hline Disseminating & Needing to report & Meeting accountability requirements \\
\hline
\end{tabular}




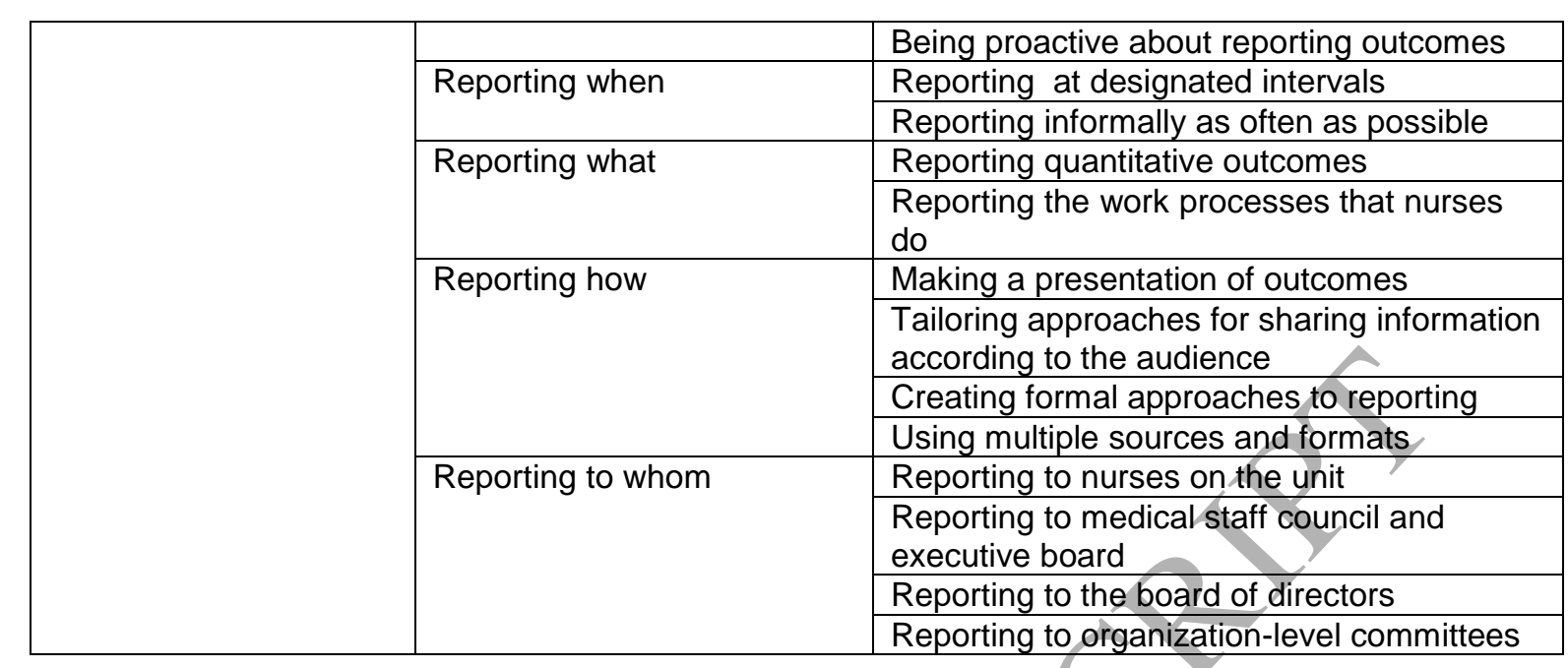

